\title{
Research Article \\ Exact Explicit Traveling Wave Solution for the Generalized (2+1)-Dimensional Boussinesq Equation
}

\section{Libing Zeng, Keding Qin, and Shengqiang Tang}

School of Mathematics and Computing Science, Guilin University of Electronic Technology, Guangxi, Guilin 541004, China

Correspondence should be addressed to Shengqiang Tang, tangsq@guet.edu.cn

Received 10 March 2011; Accepted 24 April 2011

Academic Editors: A.-C. Lee and M. Qatu

Copyright (c) 2011 Libing Zeng et al. This is an open access article distributed under the Creative Commons Attribution License, which permits unrestricted use, distribution, and reproduction in any medium, provided the original work is properly cited.

The sine-cosine method and the extended tanh method are used to construct exact solitary patterns solution and compactons solutions of the generalized $(2+1)$-dimensional Boussinesq equation. The compactons solutions and solitary patterns solutions of the generalized $(2+1)-$ dimensional Boussinesq equation are successfully obtained. These solutions may be important and of significance for the explanation of some practical physical problems. It is shown that the sinecosine and the extended tanh methods provide a powerful mathematical tool for solving great many nonlinear partial differential equations in mathematical physics.

\section{Introduction}

Studies of various physical structures of nonlinear dispersive equations had attracted much attention in connection with the important problems that arise in scientific applications. Mathematically, these physical structures have been studied by using various analytical methods, such as inverse scattering method [1], Darboux transformation method [2, 3], Hirota bilinear method [4], Lie group method [5], bifurcation method of dynamic systems [6-8], sine-cosine method [9], tanh function method [10-12], Fan-expansion method [13], and homogenous balance method [14]. Practically, there is no unified technique that can be employed to handle all types of nonlinear differential equations.

Recently, by using the sine-cosine method, Taşcan and Bekir [15] studied the following (2+1)-dimensional Boussinesq equation:

$$
u_{t t}-u_{x x}-u_{y y}-\left(u^{2}\right)_{x x}-u_{x x x x}=0
$$


More new double periodic and multiple soliton solutions are obtained for the generalized $(2+1)$-dimensional Boussinesq equation in [16]. Chen et al. [17] study (2+1)-dimensional Boussinesq equation by using the new generalized transformation in homogeneous balance method. Feng et al. [8] have investigated the bifurcations and global dynamic behavior of two variants of $(2+1)$-dimensional Boussinesq-type equations with positive and negative exponents and obtained the sufficient conditions under which solitary, kink, breaking, and periodic wave solutions appear. A mathematical method is constructed to study two variants of the two-dimensional Boussinesq water equation with positive and negative exponents in [18]. equation:

In this paper, we consider the following generalized (2+1)-dimensional Boussinesq

$$
\left(u^{n}\right)_{t t}-\left(u^{n}\right)_{x x}-\left(u^{n}\right)_{y y}-a\left(u^{2}\right)_{x x}-b\left(u^{n}\right)_{x x x x}=0
$$

where $a, b$ are arbitrary constants and $n$ is nonzero integers. Specially, when $a=b=n=1$ system (1.2) becomes the (2+1)-dimensional Boussinesq equation.

The sine-cosine method and the tanh method will be mainly used to back up our analysis. The tanh method, developed by Malfliet and Hereman [11, 12] and Wazwaz [19], is a direct and effective algebraic method for handling many nonlinear equations. The sinecosine method was proved to be powerful in handling nonlinear problems, with genuine nonlinear dispersion, where compactons and solitary patterns solutions are generated. The two methods will be described briefly, where details can be found in [9-12, 19] and the references therein.

Let $u(x, y, t)=u(\xi), \xi=x+y-c t$, where $c$ is the wave speed. Then (1.2) becomes

$$
\left(c^{2}-2\right)\left(u^{n}\right)^{\prime \prime}-a\left(u^{2}\right)^{\prime \prime}-b\left(u^{n}\right)^{\prime \prime \prime \prime}=0
$$

where "'!' is the derivative with respect to $\xi$. Integrating (1.3) twice and neglecting constants of integration, we find

$$
\left(c^{2}-2\right) u^{n}-a u^{2}-b\left(u^{n}\right)^{\prime \prime}=0
$$

Suppose that $u(\xi)$ is a continuous solution of (1.4) for $\xi \in(-\infty, \infty)$ and $\lim _{\xi \rightarrow \infty} \phi(\xi)=$ $a, \lim _{\xi \rightarrow-\infty} \phi(\xi)=b$. Recall that (i) $u(x, t)$ is called a solitary wave solution if $a=b$ and (ii) $u(x, t)$ is called a kink or antikink solution if $a \neq b$. It is well known that a limit boundary curve of a smooth family of periodic orbits gives rise to a smooth periodic traveling wave solution, that is called a compacton.

The paper is organized as follows. In Section 2, the sine-cosine method and the tanh method are briefly discussed. Section 3 represents exact analytical solutions of (1.2) by using the sine-cosine method. Section 4 represents exact analytical solutions of (1.2) by using the extended tanh method. In the last section, we conclude the paper and give some discussions. 


\section{Analysis of the Two Methods}

The sine-cosine method and the extended tanh method have been applied for a wide variety of nonlinear problems. The main features of the two methods will be reviewed briefly.

For both methods, we first use the wave variable $\xi=x+y-c t$ to carry a PDE in two independent variables

$$
P\left(u, u_{t}, u_{x}, u_{y}, u_{x x}, u_{x y}, u_{y y}, \ldots\right)=0
$$

into an ODE

$$
Q\left(u, u^{\prime}, u^{\prime \prime}, u^{\prime \prime \prime}, \ldots\right)=0
$$

Equation (2.2) is then integrated as long as all terms contain derivatives where integration constants are considered zeros.

\subsection{The Sine-Cosine Method}

The sine-cosine method admits the use of the solution in the form

$$
u(x, y, t)= \begin{cases}\lambda \cos ^{\beta}(\mu \xi), & |\mu \xi|<\frac{\pi}{2} \\ 0, & \text { otherwise }\end{cases}
$$

or in the form

$$
u(x, y, t)= \begin{cases}\lambda \sin ^{\beta}(\mu \xi), & |\mu \xi|<\pi \\ 0, & \text { otherwise }\end{cases}
$$

where $\lambda, \mu$, and $\beta$ are parameters that will be determined.

We substitute (2.3) or (2.4) into the reduced ordinary differential equation obtained above in (2.2), balance the terms of the cosine functions when (2.3) is used or balance the terms of the sine functions when (2.4) is used, and solve the resulting system of algebraic equations by using the computerized symbolic calculations to obtain all possible values of the parameters $\lambda, \mu$, and $\beta$.

\subsection{The Tanh Method and the Extended Tanh Method}

The standard tanh method is introduced in $[11,12]$ where the tanh is used as a new variable, since all derivatives of a tanh are represented by a tanh itself. We use a new independent variable

$$
Y=\tanh (\mu \xi)
$$


that leads to the change of derivatives:

$$
\begin{gathered}
\frac{d}{d \xi}=\mu\left(1-Y^{2}\right) \frac{d}{d Y^{\prime}} \\
\frac{d^{2}}{d \xi^{2}}=\mu^{2}\left(1-Y^{2}\right)\left(-2 Y \frac{d}{d Y}+\left(1-Y^{2}\right) \frac{d^{2}}{d Y^{2}}\right) .
\end{gathered}
$$

We then apply the following finite expansion:

$$
\begin{gathered}
u(\mu \xi)=S(Y)=\sum_{k=0}^{M} a_{k} Y^{k}, \\
u(\mu \xi)=S(Y)=\sum_{k=0}^{M} a_{k} Y^{k}+\sum_{k=1}^{M} b_{k} Y^{-k},
\end{gathered}
$$

where $M$ is a positive integer that will be determined to derive a closed form analytic solution. However, if $M$ is not an integer, then a transformation formula is usually used. Substituting (2.5) and (2.6) into the simplified ODE (2.2) results in an equation in powers of $Y$. To determine the parameter $M$, we usually balance the linear terms of highest order in the resulting equation with the highest order nonlinear terms. With $M$ determined, we collect all coefficients of powers of $Y$ in the resulting equation where these coefficients have to vanish. This will give a system of algebraic equations involving the parameters $a_{k}(k=0, \ldots, M), \mu$, and $c$. Having determined these parameters, knowing that $M$ is a positive integer in most cases and using (2.7) or (2.8), we obtain an analytic solution $u(x, t)$ in a closed form.

\section{Using the Sine-Cosine Method}

Substituting (2.3) into (1.4) yields

$$
-a \lambda^{2} \cos ^{2 \beta}(\mu \xi)+\left(c^{2}-2+b n^{2} \mu^{2} \beta^{2}\right) \lambda^{n} \cos ^{n \beta}(\mu \xi)-b n \mu^{2} \lambda^{n} \beta(n \beta-1) \cos ^{n \beta-2}(\mu \xi)=0 .
$$

Equation (3.1) is satisfied only if the following system of algebraic equations holds:

$$
n \beta \neq 1, \quad n \beta-2=2 \beta, \quad c^{2}+b n^{2} \mu^{2} \beta^{2}=2, \quad-a \lambda^{2}=b n \mu^{2} \lambda^{n} \beta(n \beta-1)
$$

or

$$
\lambda=\lambda \neq 0, \quad a=0, \quad b \neq 0, \quad n \beta-1=0, \quad c^{2}+b n^{2} \mu^{2} \beta^{2}=2 .
$$


Solving the system (3.2) and (3.3) gives

$$
\begin{gathered}
\beta=\frac{2}{n-2}, \quad \mu^{2}=\frac{\left(2-c^{2}\right)(n-2)^{2}}{4 b n^{2}}, \quad \lambda=\left[\frac{2 a n}{\left(c^{2}-2\right)(n+2)}\right]^{1 / n-2}, \quad n \neq \pm 2, a b \neq 0, \\
b \neq 0, \quad \lambda=\lambda \neq 0, \quad a=0, \quad \beta=\frac{1}{n}, \quad \mu^{2}=\frac{2-c^{2}}{b} .
\end{gathered}
$$

The results (3.4) and (3.5) can be easily obtained if we also use the sine method (2.4). Combining (3.4) and (3.5) with (2.3) and (2.4), the following compactons solutions are yield:

$u_{1}= \begin{cases} \pm\left[\frac{2 a n}{\left(c^{2}-2\right)(n+2)} \cos ^{2} \frac{n-2}{2 n} \sqrt{\frac{2-c^{2}}{b}}(x+y-c t),\right]^{1 / n-2}, \\ |x+y-c t|<\frac{n \pi}{n-2} \sqrt{\frac{b}{2-c^{2}}}, \quad n= \pm 2(k+1), \quad k \in Z^{+}, b\left(2-c^{2}\right)>0, a\left(c^{2}-2\right)>0, \\ 0, & \text { otherwise, }\end{cases}$

$u_{2}= \begin{cases} \pm\left[\frac{2 a n}{\left(c^{2}-2\right)(n+2)} \cos ^{2} \frac{n-2}{2 n} \sqrt{\frac{2-c^{2}}{b}}(x+y-c t)\right]^{1 / n-2}, \\ |x+y-c t|<\frac{n \pi}{n-2} \sqrt{\frac{b}{2-c^{2}}}, \quad n=2 k+1, \quad k \in Z, b\left(2-c^{2}\right)>0 \\ 0, & \text { otherwise, }\end{cases}$

$u_{3}= \begin{cases}\lambda \cos ^{1 / n} \sqrt{\frac{2-c^{2}}{b}}(x+y-c t), & \\ |x+y-c t|<\frac{\pi}{2} \sqrt{\frac{b}{2-c^{2}}}, & b\left(2-c^{2}\right)>0, \lambda \neq 0, a=0, n \neq 0, \\ 0, & \text { otherwise, }\end{cases}$

$u_{4}= \begin{cases} \pm\left[\frac{2 a n}{\left(c^{2}-2\right)(n+2)} \sin ^{2} \frac{n-2}{2 n} \sqrt{\frac{2-c^{2}}{b}}(x+y-c t)\right]^{1 / n-2}, \\ |x+y-c t|<\frac{n \pi}{n-2} \sqrt{\frac{b}{2-c^{2}}}, & n= \pm 2(k+1), k \in Z^{+}, b\left(2-c^{2}\right)>0, a\left(c^{2}-2\right)>0, \\ 0, & \text { otherwise, }\end{cases}$

$u_{5}= \begin{cases} \pm\left[\frac{2 a n}{\left(c^{2}-2\right)(n+2)} \sin ^{2} \frac{n-2}{2 n} \sqrt{\frac{2-c^{2}}{b}}(x+y-c t),\right]^{1 / n-2}, \\ |x+y-c t|<\frac{n \pi}{n-2} \sqrt{\frac{b}{2-c^{2}}}, & n=2 k+1, k \in Z, b\left(2-c^{2}\right)>0, \\ 0, & \text { otherwise, }\end{cases}$ 
$u_{6}= \begin{cases}\lambda \sin ^{1 / n} \sqrt{\frac{2-c^{2}}{b}}(x+y-c t), & b\left(2-c^{2}\right)>0, \lambda \neq 0, a=0, n \neq 0, \\ |x+y-c t|<\frac{\pi}{2} \sqrt{\frac{b}{2-c^{2}}}, & \text { otherwise }\end{cases}$

However, for $b\left(2-c^{2}\right)<0$, we obtain the following solitary patterns solutions:

$$
\begin{aligned}
& u_{7}= \pm\left[\frac{2 a n}{\left(c^{2}-2\right)(n+2)} \cosh ^{2} \frac{n-2}{2 n} \sqrt{\frac{2-c^{2}}{-b}}(x+y-c t)\right]^{1 / n-2}, n= \pm 2(k+1), k \in Z^{+}, \quad b\left(2-c^{2}\right)<0, \quad a\left(c^{2}-2\right)>0, \\
& u_{8}=\left[\frac{2 a n}{\left(c^{2}-2\right)(n+2)} \cosh ^{2} \frac{n-2}{2 n} \sqrt{\frac{2-c^{2}}{-b}}(x+y-c t),\right]^{1 / n-2}, \\
& n=2 k+1, k \in Z, \quad b\left(2-c^{2}\right)<0, \\
& u_{9}= \pm\left[\frac{-2 a n}{\left(c^{2}-2\right)(n+2)} \sinh ^{2} \frac{n-2}{2 n} \sqrt{\frac{2-c^{2}}{-b}}(x+y-c t)\right]^{1 / n-2}, \\
& n= \pm 2(k+1), k \in Z^{+}, \quad b\left(2-c^{2}\right)<0, a\left(c^{2}-2\right)<0, \\
& u_{10}=\left[\frac{-2 a n}{\left(c^{2}-2\right)(n+2)} \sinh ^{2} \frac{n-2}{2 n} \sqrt{\frac{2-c^{2}}{-b}}(x+y-c t)\right]^{1 / n-2}, \\
& n=2 k+1, k \in Z, \quad b\left(2-c^{2}\right)<0 .
\end{aligned}
$$

\section{Using the Extended Tanh Method}

In this section, we employ the extended tanh method to (1.4). Balancing $u^{2}$ with $u^{n-1} u^{\prime \prime}$, we find

$$
M=-\frac{2}{n-2} .
$$


To get a closed-form analytic solution, the parameter $M$ should be an integer. A transformation formula

$$
u=\phi^{-1 / n-2}
$$

should be used to achieve our goal. This in turn transforms (1.4) to

$$
\left(c^{2}-2\right) \phi^{2}-a \phi^{3}-\frac{2 b n^{2}}{(n-2)^{2}}\left(\phi^{\prime}\right)^{2}+\frac{b n}{n-2} \phi \phi^{\prime \prime}=0
$$

Balancing $\phi \phi^{\prime \prime}$ with $\phi^{3}$ gives $M=2$. The extended tanh method allows us to use the substitution

$$
\phi(\xi)=S(Y)=A_{0}+A_{1} Y+A_{2} Y^{2}+B_{1} Y^{-1}+B_{2} Y^{-2}
$$

Substite (4.4) into (4.3), collecte the coefficients of each power of $Y$, and solve the resulting system of algebraic equations with the help of Maple to find the sets of solutions:

$$
\begin{array}{r}
A_{1}=B_{1}=A_{2}=0, \quad B_{2}=-A_{0}, \quad A_{0}=A_{0}, \quad c^{2}=-\frac{2\left(\mathrm{a} n A_{0}-n-6+2 a A_{0}\right)}{n+6}, \\
\mu^{2}=\frac{a A_{0}(n-2)^{2}}{2 b n(n+6)}, \quad a b A_{0}(n-2)(n+6) \neq 0, \\
A_{1}=B_{1}=B_{2}=0, \quad A_{2}=-A_{0}, \quad c^{2}=-\frac{2\left(a n A_{0}-n-6+2 a A_{0}\right)}{n+6}, \\
\mu^{2}=\frac{a A_{0}(n-2)^{2}}{2 b n(n+6)}, \quad a b A_{0}(n-2)(n+6) \neq 0, \\
A_{1}=B_{1}=0, \quad A_{0}=A_{0}, \quad B_{2}=-\frac{1}{2} A_{0}, \quad c^{2}=-\frac{2\left(2 a n A_{0}-n-6+4 a A_{0}\right)}{n+6}, \\
\mu^{2}=\frac{a A_{0}(n-2)^{2}}{4 b n(n+6)}, \quad a b A_{0}(n-2)(n+6) \neq 0 .
\end{array}
$$

We obtain the solitary wave solution and the solitary patterns solutions

$$
\begin{aligned}
& u_{11}=\left[\frac{\sinh ^{2}\left((n-2) \sqrt{a A_{0} /(2 b n(n+6))}(x+y-c t)\right)}{-A_{0}}\right]^{1 / n-2}, \quad A_{0}<0, \quad a b n(n+6)<0, \\
& c^{2}=-\frac{2\left(a n A_{0}-n-6+2 a A_{0}\right)}{n+6}>0, \quad n= \pm 2 k, k \in Z^{+}, n \neq 2,
\end{aligned}
$$




$$
\begin{aligned}
& u_{12}=\left[\frac{\sinh ^{2}\left((n-2) \sqrt{a A_{0} /(2 b n(n+6))}(x+y-c t)\right)}{-A_{0}}\right]^{1 / n-2}, \quad a b n(n+6) A_{0}>0, \\
& c^{2}=-\frac{2\left(a n A_{0}-n-6+2 a A_{0}\right)}{n+6}>0, \quad n=2 k+1, k \in Z, \\
& u_{13}= \pm\left[\frac{\cosh ^{2}\left((n-2) \sqrt{a A_{0} /(2 b n(n+6))}(x+y-c t)\right)}{A_{0}}\right]^{1 / n-2}, \quad A_{0}>0, a b n(n+6)<0, \\
& c^{2}=-\frac{2\left(a n A_{0}-n-6+2 a A_{0}\right)}{n+6}>0, \quad n= \pm 2 k, k \in Z^{+}, n \neq 2, \\
& u_{14}=\left[\frac{\cosh ^{2}\left((n-2) \sqrt{a A_{0} /(2 b n(n+6))}(x+y-c t)\right)}{A_{0}}\right]^{1 / n-2}, \quad a b n(n+6) A_{0}>0, \\
& c^{2}=-\frac{2\left(a n A_{0}-n-6+2 a A_{0}\right)}{n+6}>0, \quad n=2 k+1, k \in Z, \\
& c^{2}=-\frac{2\left(2 a n A_{0}-n-6+4 a A_{0}\right)}{n+6}>0, \quad n=2 k+1, k \in Z . \\
& u_{15}= \pm\left[\frac{\sinh ^{2}\left((n-2) \sqrt{a A_{0} /(b n(n+6))}(x+y-c t)\right)}{-2 A_{0}}\right]^{1 / n-2}, \quad A_{0}<0, a b n(n+6)<0, \\
& c_{16}^{2}=-\frac{2\left(2 a n A_{0}-n-6+4 a A_{0}\right)}{n+6}>0, \quad n= \pm 2 k, k \in Z^{+}, n \neq 2, \\
& {\left[\frac{\sinh ^{2}\left((n-2) \sqrt{a A_{0} /(b n(n+6))}(x+y-c t)\right)}{n-n-2}, a b n(n+6) A_{0}>0,\right.}
\end{aligned}
$$

We can obtain the following compactons solutions for (1.4):

$$
\begin{gathered}
u_{17}= \pm\left[\frac{\sin ^{2}\left((n-2) \sqrt{-a A_{0} /(2 b n(n+6))}(x+y-c t)\right)}{A_{0}}\right]^{1 / n-2}, \quad A_{0}>0, a b n(n+6)<0, \\
c^{2}=-\frac{2\left(a n A_{0}-n-6+2 a A_{0}\right)}{n+6}>0, \quad n= \pm 2 k, k \in Z^{+}, n \neq 2, \\
u_{18}=\left[\frac{\sin ^{2}\left((n-2) \sqrt{-a A_{0} /(2 b n(n+6))}(x+y-c t)\right)}{A_{0}}\right]^{1 / n-2}, \quad A_{0}>0, a b n(n+6)<0, \\
c^{2}=-\frac{2\left(a n A_{0}-n-6+2 a A_{0}\right)}{n+6}>0, \quad n=2 k+1, k \in Z,
\end{gathered}
$$




$$
\begin{aligned}
& u_{19}= \pm\left[\frac{\cos ^{2}\left((n-2) \sqrt{-a A_{0} /(2 b n(n+6))}(x+y-c t)\right)}{A_{0}}\right]^{1 / n-2}, \quad A_{0}>0, a b n(n+6)<0 \text {, } \\
& c^{2}=-\frac{2\left(a n A_{0}-n-6+2 a A_{0}\right)}{n+6}>0, \quad n= \pm 2 k, k \in Z^{+}, n \neq 2, \\
& u_{20}=\left[\frac{\cos ^{2}\left((n-2) \sqrt{-a A_{0} /(2 b n(n+6))}(x+y-c t)\right)}{A_{0}}\right]^{1 / n-2}, \quad A_{0}>0, a b n(n+6)<0 \text {, } \\
& c^{2}=-\frac{2\left(a n A_{0}-n-6+2 a A_{0}\right)}{n+6}>0, \quad n=2 k+1, k \in Z, \\
& u_{21}= \pm\left[\frac{\sin ^{2}\left((n-2) \sqrt{-a A_{0} /(b n(n+6))}(x+y-c t)\right)}{2 A_{0}}\right]^{1 / n-2}, \quad A_{0}>0, a b n(n+6)<0 \text {, } \\
& c^{2}=-\frac{2\left(2 a n A_{0}-n-6+4 a A_{0}\right)}{n+6}>0, \quad n= \pm 2 k, k \in Z^{+}, n \neq 2, \\
& \begin{array}{c}
u_{22}= \pm\left[\frac{\sin ^{2}\left((n-2) \sqrt{-a A_{0} /(b n(n+6))}(x+y-c t)\right)}{2 A_{0}}\right]^{1 / n-2}, \quad A_{0}>0, a b n(n+6)<0, \\
c^{2}=-\frac{2\left(2 a n A_{0}-n-6+4 a A_{0}\right)}{n+6}>0, \quad n=2 k+1, k \in Z .
\end{array}
\end{aligned}
$$

\section{Discussion}

The sine-cosine method and the extended tanh method were used to investigate the generalized (2+1)-dimensional Boussinesq equation. The study revealed compactons solutions and solitary patterns solutions for some examined variants. The study emphasized the fact that the two methods are reliable in handling nonlinear problems. The obtained results clearly demonstrate the efficiency of the two methods used in this work. Moreover, the methods are capable of greatly minimizing the size of computational work. This emphasizes the fact that the two methods are applicable to a wide variety of nonlinear problems.

\section{Acknowledgments}

The authors wish to thank the anonymous reviewers for their helpful comments and suggestions. This work is supported by the National Natural Science Foundation of China (no. 11061010).

\section{References}

[1] M. J. Ablowitz and P. A. Clarkson, Solitons, Nonlinear Evolution Equations and Inverse Scattering, vol. 149 of London Mathematical Society Lecture Note Series, Cambridge University Press, Cambridge, UK, 1991. 
[2] V. B. Matveev and M. A. Salle, Darboux Transformations and Solitons, Springer Series in Nonlinear Dynamics, Springer, Berlin, Germany, 1991.

[3] C. H. Gu, H. S. Hu, and Z. X. Zhou, Darboux Transformations in Soliton Theory and Its Geometric Applications, Shanghai Sci-Tech, Shanghai, China, 1999.

[4] R. Hirota and J. Satsuma, "Soliton solutions of a coupled KdV equation," Physics Letters. A, vol. 85, no. 8-9, pp. 407-408, 1981.

[5] P. J. Olver, Applications of Lie Groups to Differential Equations, vol. 107 of Graduate Texts in Mathematics, Springer, New York, NY, USA, 2nd edition, 1993.

[6] J. B. Li and Z. Liu, "Travelling wave solutions for a class of nonlinear dispersive equations," Chinese Annals of Mathematics, vol. 23B, no. 3, pp. 397-418, 2002.

[7] S. Tang and W. Huang, "Bifurcations of travelling wave solutions for the generalized double sinhGordon equation," Applied Mathematics and Computation, vol. 189, no. 2, pp. 1774-1781, 2007.

[8] D. Feng, T. He, and J. Lü, "Bifurcations of travelling wave solutions for (2+1)-dimensional Boussinesqtype equation," Applied Mathematics E Computation, vol. 185, no. 1, pp. 402-414, 2007.

[9] S. Tang, Y. Xiao, and Z. Wang, "Travelling wave solutions for a class of nonlinear fourth order variant of a generalized Camassa-Holm equation," Applied Mathematics $\mathcal{E}$ Computation, vol. 210, no. 1, pp. 39-47, 2009.

[10] S. Tang, J. Zheng, and W. Huang, "Travelling wave solutions for a class of generalized KdV equation," Applied Mathematics \& Computation, vol. 215, no. 7, pp. 2768-2774, 2009.

[11] W. Malfliet and W. Hereman, "The tanh method: I. Exact solutions of nonlinear evolution and wave equations," Physica Scripta, vol. 54, no. 6, pp. 563-568, 1996.

[12] W. Malfliet and W. Hereman, "The tanh method. II. Perturbation technique for conservative systems," Physica Scripta, vol. 54, no. 6, pp. 569-575, 1996.

[13] E. Fan, "Uniformly constructing a series of explicit exact solutions to nonlinear equations in mathematical physics," Chaos, Solitons E Fractals, vol. 16, no. 5, pp. 819-839, 2003.

[14] M. L. Wang, "Exact solutions for a compound KdV-Burgers equation," Physics Letters. A, vol. 213, no. 5-6, pp. 279-287, 1996.

[15] F. Taşcan and A. Bekir, "Analytic solutions of the (2+1)-dimensional nonlinear evolution equations using the sine-cosine method," Applied Mathematics \& Computation, vol. 215, no. 8, pp. 3134-3139, 2009.

[16] H.-T. Chen and H.-Q. Zhang, "New double periodic and multiple soliton solutions of the generalized (2+1)-dimensional Boussinesq equation," Chaos, Solitons E Fractals, vol. 20, no. 4, pp. 765-769, 2004.

[17] Y. Chen, Z. Yan, and H-Q. Zhang, "New explicit solitary wave solutions for (2+1)-dimensional Boussinesq equation and (3+1)-dimensional KP equation," Physics Letters. A, vol. 307, no. 2-3, pp. 107-113, 2003.

[18] S. Lai, Y-H. Wu, and Y. Zhou, "Some physical structures for the (2+1)-dimensional Boussinesq water equation with positive and negative exponents," Computers $\mathcal{E}$ Mathematics with Applications, vol. 56, no. 2, pp. 339-345, 2008.

[19] A. M. Wazwaz, "A class of nonlinear fourth order variant of a generalized Camassa-Holm equation with compact and noncompact solutions," Applied Mathematics E Computation, vol. 165, no. 2, pp. 485-501, 2005. 


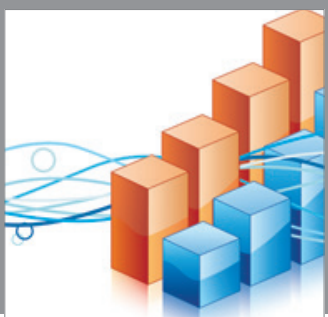

Advances in

Operations Research

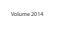

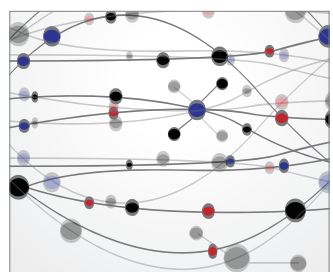

\section{The Scientific} World Journal
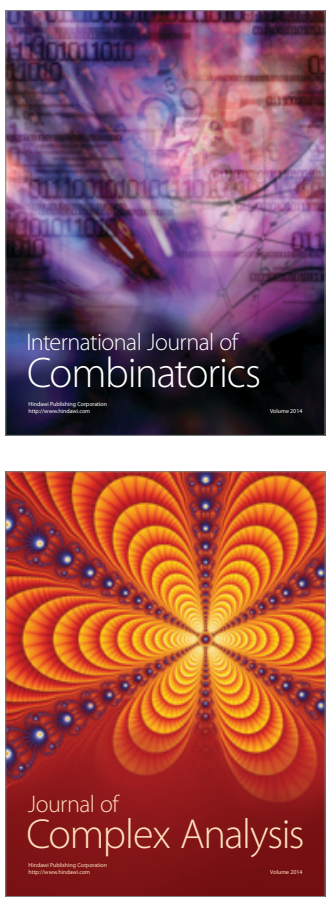

International Journal of

Mathematics and

Mathematical

Sciences
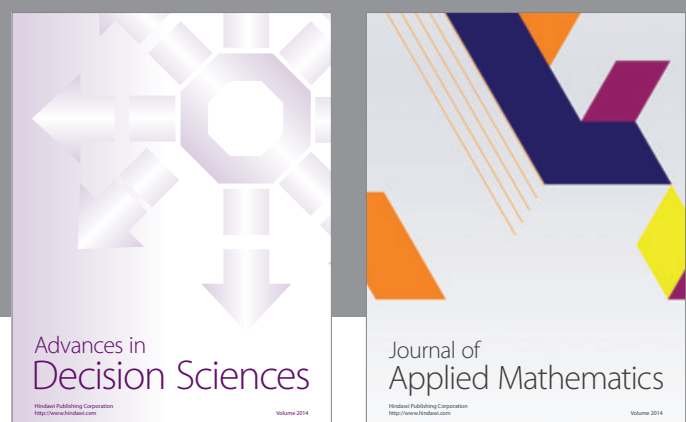

Journal of

Applied Mathematics
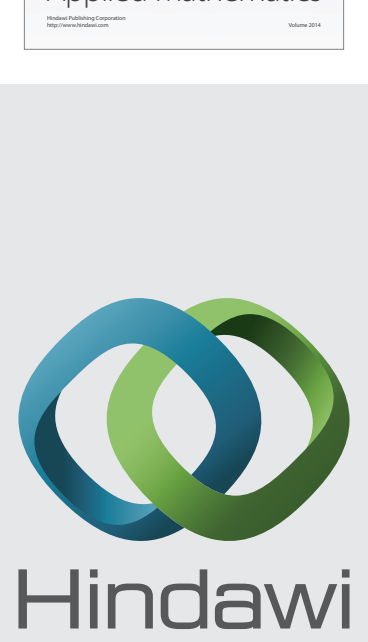

Submit your manuscripts at http://www.hindawi.com
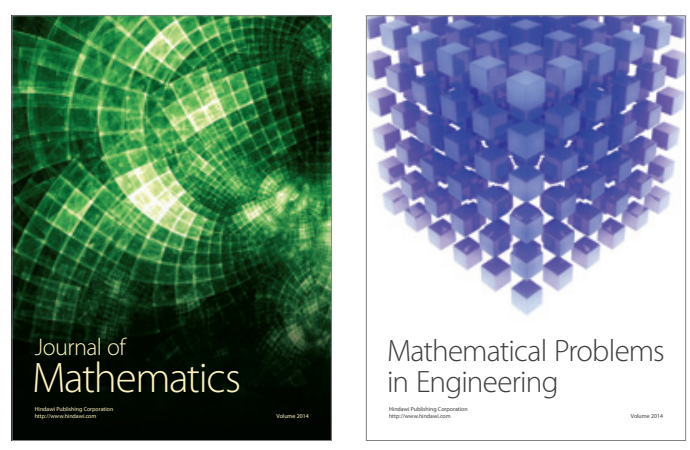

Mathematical Problems in Engineering
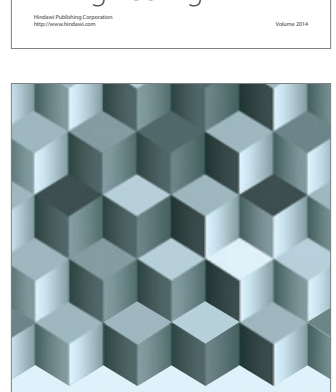

Journal of

Function Spaces
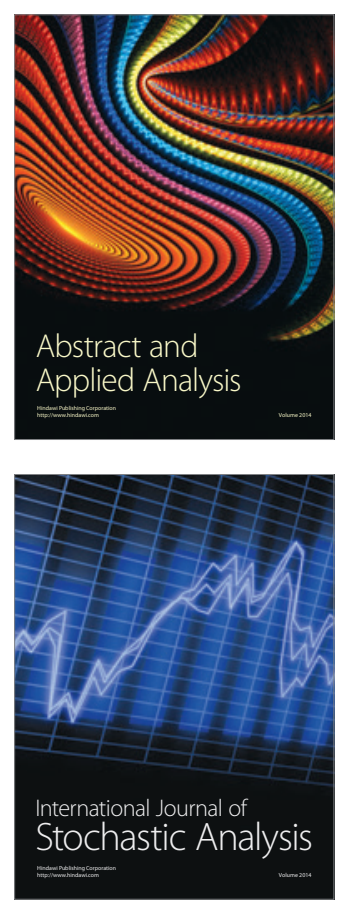

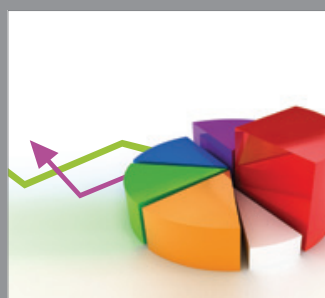

ournal of

Probability and Statistics

Promensencen
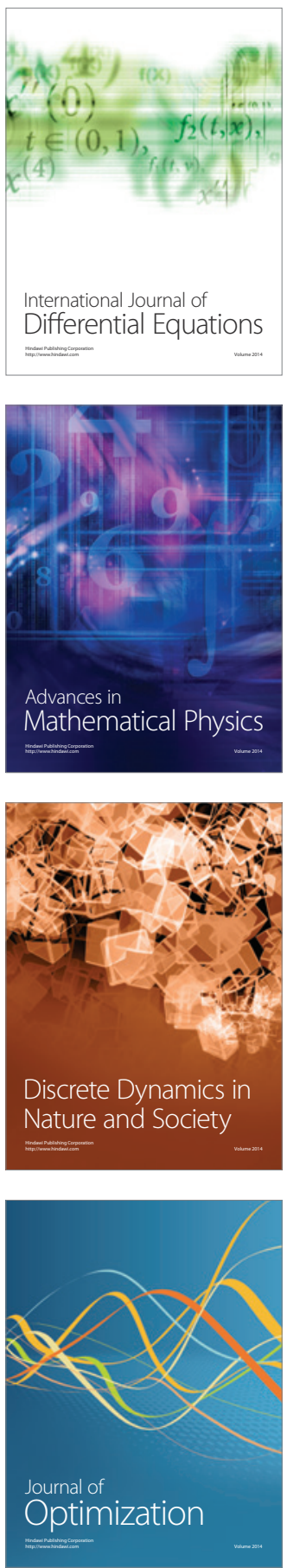